Journal of the American Medical Association.

EDITED FOR THE ASSOCIATION BY N. S. DAVIS.

PUBLISHED WEEKLY.

Vol. VIII.

Chicago, April 2, 1887.

No. I 4 .

ORIGINAL ARTICLES.

HYSTERO-NEURASTHENIA, OR NERVOUS EXHAUSTION OF WOMEN, TREATED BY THE S. WEIR MITCHELL METHOD.

Read before the Chicago Medical Society, March 7, 1887 . BY FRANKLIN H. MARTIN, M.D.,

PROFESSOR OF GYNECOLOGY IN THE CHICAGO POLICLINIC; FELLOW OF CHICAGO GYNECOLOGICAL SOCIETY.

Hystero-neurasthenia is a name that I will take the liberty of giving to an often recognized class of female difficulties which has not a well defined place in medical literature.

The symptoms of this class of cases are as difficult to enumerate and describe as the individual cases are difficult to manage to a successful issue. Under the term hystero-neurasthenia, I wish to include no symptoms which can be traced to a distinct pathological lesion of any one organ, but to a host of symptoms that can be accounted for in no other manner than by being the result of a partial or general nervous inefficiency, or perversion, of the nerves controlling the organs peculiar to women.

The first of these conditions, nervous inefficiency, may be congenital, or the result of excessive exercise of the functions of the organs of the pelvis, from a long and prolific child-bearing season, excessive cohabitation, or undue treatment of a local variety. The second condition, nervous perversion, will be found the result of excessive brain work, either as a consequence of early study, or from literary excess, teaching, and clerical work common to women of maturer life, the worries of motherhood, anxieties of impending or actual misfortune, prolonged lactation, nursing of the sick, excessive physical labor, and, rarely, masturbation.

The symptoms of this class of cases are too numerous to mention in detail, and inasmuch as each case has its own peculiarities, I will remain content to recite a few of the most prominent and common symptoms. General anæmia, or deficiency of red corpuscles, is a very common, but not universal symptom. The anremic cases usually complain of loss of flesh, although it is well known some anamic patients gain flesh; and where this state of affairs exists the functions of the generative organs usually suffer, as is often manifested by coincident amenorrhœa and ster. ility. Upon questioning these patients the key-note of a general outpouring of subjective symptoms is struck, when the womb is reached in the list of interrogations-bearing-down pains, backache, leucorrhcea, neuralgia in ovarian region, painful menstruation (pain before menstruation, during the flow, and for days following), frequent urination, constipation"bowels never move without medicine"-painful defecation, neuralgia in all parts of pelvis at regular or jrregular intervals, in the ovarian region, one or both sides, uterus, vagina, bladder, perineum, rectum, and even the urethra. Standing and walking is accomplished very seldom without fatigue, and scarcely less rarely without pain in the loins and lower pelvic region.

These sufferers usually have worn out the patience of one or two physicians; many times are pronounced hysterical incurables, who imagine many of their aches, and are therefore considered unworthy of more dignified attention than that required to prescribe an anodyne, a hypnotic or a blister. These cases are much too interesting, and the credit of effecting a permanent cure of too much gratification, to say nothing of duty, for us to be satisfied to listlessly alleviate symptoms, when it lies in our power to do more.

Upon physical examination this class of patients present few well-marked subjective symptoms. There is no evidence of hereditary taints. As this trouble is referred to the uterus, the first local examination is made of the pelvic organs. The vulva is often pale in color from general anæmia. The mucous membrane within the vagina presents a faded, lax appearance. The external genitals are sometimes bathed with mucus secretion from the relaxed vulvo vaginal glands. 'The vagina is often sensitive, bathed with mucus frequently; at other times abnormally free from secretion. The uterus is normal in size and location, with perhaps the exception of slight prolapsus from the general relaxation of all the surrounding parts. It is very movable, as a rule. The organ is very liable to be marked by hypersensitiveness on pressure. A slight mucus discharge from the cervix, of a milky character, is rarely absent. The cervix is usually pale in color. Pressure in the ovarian region causes pain, or at least a feeling of sensitiveness; the ovaries can be frequently engaged between the hand placed over the relaxed, thin abdominal walls, and the index finger in the vagina, and will often be found considerably enlarged. The rectum is rarely found in other than a relaxed condition. There is frequently much tenderness about the anus, with slight nodular enlargement of the external hæmor- 
rhoidal veins. Fæces are often found in the lower bowel. The bladder is often sensitive to slight pressure, and not rarely, where the patient is anæmic, will there be a pouching of its neck. The urine is pale, and commonly filled with phosphates and mucus. Upon manipulating the abdominal wall there is scarcely a point that does not seem most sensitive, except, perhaps, the ovarian region. The muscles are flabby and relaxed upon the limbs, although occasionally covered with soft fat.

The heart action in these cases, while not strong, is usually regular. The exception to this is in very nervous patients where, through. sympathy of a rebellious or weak stomach, palpitation and heart-burn will be a complication. The capillary circulation is frequently slow, as evidenced by the slow return of the circulation to a part deprived of its blood by pressure of the finger.

The digestion is often fair, but rarely very good. Attacks of nausea are frequently complained of, coming on without any warning and disappearing in the same way. Occasionally downright attacks of indigestion are experienced, which may not be confined to the stomach, but affect the whole digestive tract. These attacks may be accompanied with headache. Spinal tenderness in one or more regions is often present; this will frequently be found quite marked in the lower dorsal or first lumbar region.

Besides the symptoms enumerated, which can be said to rank under the head of hystero-neurasthenia, we may have, in addition, all the symptoms that are common to general neurasthenia, the special symptoms about the pelvis determining the disease, because of their greater prominence and severity. While we have found by general examination of these cases, both subjective and objective, not one organ in the pelvis or abdomen in vigorous health, and not one free from weakness and tenderness, we have not found an actually diseased member - that is, diseased from any pathological condition peculiar to itself, but rather from a general lack of balance between supply and demand in the nutrition of several. And while it is rare for a single case to present all the symptoms above noted, occasionally, as many of us can testify, all these and others will manifest themselves in their most aggravating form in one suffering individual.

Though no exact pathology of these cases has been definitely demonstrated, except through the deductions drawn from successful treatment, the fault is generally conceded to lie in a weakened or incomplete state of that part of the nervous system which presides over the nutrition of the organs involved. A general malnutrition, then, of the parts implicated is that towards which we must direct our treatment. As these patients always complain of being tired, the first indication for treatment is rest. As they are almost invariably anæmic, proper feeding is the second indication. As a case is rarely found in which nervous debility is not the rule, seclusion from annoying surroundings is a third indication. Sleeplessness, which is frequently a conspicuous symptom, gives us a fourth indication.

The prominent requirements, then, in these cases are: I. Rest. 2. Proper Feeding. 3. Seclusion. 4. Sleep.
How can we obtain these four requisites without over drugging our patients? We must introduce some means by which an írritable body, that is unable to assume the recumbent position without resting upon some painful spot, may lie down without: pain. We must feed properly a patient whose appetite is capricious, whose stomach may be irritable and rebellious, whose bowels will not "agree" with anything that is suitable to sustain life. We must put into seclusion patients who imagine they require the sustaining sympathy of innumerable dear ones. We must produce sleep in a class of patients who have long ago worn out all the safe and efficient hypnotics.

I have had experience enough with these cases to satisfy myself that permanent cures can very often be effected by a line of treatment that has been practised so successfully in general neurasthenia by that eminent Philadelphian, S. Weir Mitchell. While Dr. Mitchell was the originator of this systematic line of treatment, of which I can only hope to give merely an outline this evening, it has been adopted with great success by others, and by none with greater success than Playfair, of London.

Dr. Mitchell seeks to meet the four requirements. in the treatment of these cases by first getting full control and confidence of the patient. Without this. first requisite, the case is a failure. After this is accomplished he makes the remaining part of the problem feasible by a combination of entire rest and of excessive feeding made possible by passive txercise obtained through the use of massage and electricity.

A physician, to treat these cases successfully, must have an eye to detail, possess at least the ordinary amount of tact, perseverance, firmness, and good executive ability. The nurses employed should be educated, intelligent, strong young persons, who are able and willing to work, and who can make themselves very agreeable; who possess tact and firmness, the latter without sternness. They should understand and be capable of performing thorough massage, administer a vaginal douche properly, and be adepts at preparing tempting sick-room delicacies.

In further describing this system of treatment I will give, for the sake of brevity, the details of treatment of a typical case of the kind that recently came under my observation:

The patient, a young married lady in the better class of society, without children, had been treated for "womb difficulties" for three years by at least two St. Louis physicians, from which city she had recently moved to Chicago. She had gradually become worn out from unsuccessful local treatment, and was about to give up in despair. She was in an extreme state of anæmia; had been gradually reduced in flesh from $\mathrm{t} 20$ to roo lbs. Menstruation irregular and painful throughout. Bowels never moved without assistance. Appetite gone, and what little food she could be induced to take remained like a load on her stomach and gave her considerable pain. Besides the loss of appetite and indigestion, she had considerable ovarian neuralgia, general pelvic hyperæsthesia, and intense sa.cralgia. She was also troubled with persistent insomnia.

Physical examination elicited no localized patho- 
logical condition. Uterus natural in size and location, and movable. Ovaries not enlarged, but very tender. While there was general hyperæsthesia in every direction from the vagina, there evidently was no cellulitis or peritoneal intlammation. The urine was normal.

The scheme of treatment mentioned above was explained to the patient, and she immediately acquiesced when advised to make a trial of it. Contrary to Dr. Mitchell's advice, she was not separated from her family, there being but her husband, and he at home but a small portion of the day. Be it remembered, she was unable to retain even a very little of the blandest food without distress.

She was immediately put upon an exclusive milk diet. The diet for the first day was laid down as three ounces of milk every three hours. She took at this rate, in the first twenty-four hours, twenty-one ounces of milk. The second day the amount was increased to twenty-eight ounces by increasing each allowance to four ounces.

The third day, as the patient was doing remarkably well and the stomach was free from pain, one ounce of thinly cut stale bread, well toasted, was given in addition to the milk that was due at the three regular meal times.

The fourth day six ounces of milk were taken every two hours, with double the amount of toast that was given the day before. All the dyspeptic symptoms had at this time disappeared, and the patient, notwithstanding the amount of milk taken, commenced to ask for her meals.

The fifth day she was allowed in addition to the milk, for breakfast, about one and one-half ounces of finely chopped steak of beef rarely broiled; this, with about one ounce of stale bread with butter, was taken with great relish, and without subsequent distress. At noon, on account of a little feeling of nausea, she had nothing except her regular milk diet. An afternoon sleep left her with an appetite for her supper. She was then given three or four raw oysters, with toast and a small cup of tea. This was taken with relish, and there was a disposition to take more.

The sixth day, on account of the patient exhibiting a slight disgust for the large doses of milk, the allowance was reduced to four ounces, it being rendered more nutritious by making it one third cream. Besides the twenty-eight ounces of milk and cream, she was given this day the juice from one pound of beef in three doses, at IO A.M. and 3 and 8 P.M. Besides this, the patient took part of a cup of coffee and an ounce of bread with butter for her breakfast, a lamb chop for her dinner, with bread and butter, and raw oysters with toasted crackers and butter for 6 o'clock supper.

From this time on while the patient remained under treatment with me, she had no trouble, with judicious care in its selection, in retaining and relishing an incredibly large amount of food. She would take, besides three large meals a day, a quart of milk and cream, and the juice from one-half to one pound of beef.

The digestion and assimilation of this large quantity of food by an irritable alimentary canal was

made possible by systematic passive exercise. The routine of treatment for the day in this case was as follows: At 8:30 A.M., or as soon as the patient had awakened, she was given a light sponge bath, her hair was arrangeci, and her milk and breakfast taken. At 9:30 A.M., if the bowels had not moved spontaneously, a small rectal injection of soap and water was administered. This was not found necessary after a few days' treatment. At II A.M. or thereabouts the patient was given general faradization with an idea of reaching all the motor points of the superficial muscles. The region of the colon, especially of the transverse and descending colon, with special efforts at stimulating the rectum, was systematically sought. This treatment required from three-fourths to one hour's time. The patient usually took a short nap after this treatment. About I o'clock she was induced to take a light dinner, or, more properly, lunch. In the afternoon, if necessary, the nurse would interest the patient by light reading for an hour, if she were not inclined to sleep, which was frequently the case. At 5 o'clock a light, rapid sponge bath was administered; followed by gentle rubbing of skin with a dry bath glove. This proceeding occupied thirty minutes, after which the patient was again allowed to rest for an hour. At $6: 30$ or 7 P.M. she was given her dinner. The milk, in the meantime, had been administered at regular intervals throughout the day. After the dinner or supper the patient was read to or amused in some way, or, if she was so inclined, allowed to sleep. Nothing, except the amount they eat, will astonish one more than the amount of sleep some of these patients seem to require.

At $8: 30$ or 9 P.M. the regular preparation for bed commenced. This began with a systematic massage which included all parts of the external muscular system, and occupied about one hour. The patient was then moved to a couch, given a large vaginal douche of hot water while in the recumbent position; her bed in the meantime was changed, and she was at last, after a hard day's work in which she had been but a passive laborer, deposited in it for the night.

This patient remained under this systematic treatment for about eighteen days only, at the end of which time it did not seem necessary that she should be kept under such close observation longer. She expressed a very strong desire to go with her husband, who was about to make a business trip South. Inasmuch as she was, to all appearances, now perfectly well, I gave my consent to this arrangement. She could take and digest more food than she had been able to for years, without a dyspeptic symptom; her menstrual period had passed without a pain; the pelvic hyperæsthesia, while not entirely subdued, was much improved; the vaginal leucorrhoea was entirely checked. The patient had gained ten pounds in weight. Her skin was now ruddy and healthy in appearance, and she felt strong, well, and in the best of spirits when she left the city. I have since seen the patient, and she assures me that she is in the best of health, and she certainly appears so.

All cases, however, in which the treatment described here seems applicable, will not give the biilliant results that twenty days' treatment accomplished 
for me in the above case. Every symptom of importance here disappeared after four days' treatment, and subsequently there was nothing left to accomplish but to increase the flesh and strength of the patient. The getting-up was gradual; at the end of about the ninth day she was allowed to sit up in a large upholstered armchair for one hour in the forenoon. This was followed rapidly with greater liberty, and at the end of the fifteenth day she was about the room fully dressed; and at the end of the twentieth day was ready to travel.

Frequently grave complications are met in the treatment of these cases. Occasionally a patient has been thoroughly disgusted with milk on account of excessive use of it in previous treatments. Others have the impression that it "makes them bilious," and it is not taken on that account. By taking pains to explain that milk is one of the most perfect forms of food, and that it is an important factor in the successful treatment, most patients will be induced to try it in small, often repeated doses. There is occasionally a patient found, however, who cannot take milk in the raw state; the taste is objectionable, and the stomach rejects it. In such cases milk will often be well received if prepared with Fairchild Bros. \& Foster's peptogenic milk powder. This is often also a valuable addition when the patient becomes tired of milk late in the treatment. Beef juice prepared after Weir Mitchell's formula, either raw or cooked, is sometimes a good substitute. Patients are frequently found who can take scalded milk who cannot bear it in the raw state; and, again, frequently if mixed with cream, when milk alone nauseates. Where this important article of diet is not tolerated under any disguise, other food must be adopted which will accomplish the same end. Here, in selecting a suitable substitute for milk from the long list of natural and artificial foods, is where the ingenuity and experience of the physician are heavily taxed.

Occasionally among these patients will be found one who requires an alcoholic stimulant. This is often indicated where there are sudden attacks of nausea. Hoff's fluid malt often arouses a desire for food, if given three or four times a day in small doses; the hop principle often acting, in addition, as a pleasant hypnotic in these cases.

Where the bowels are not sufficiently stimulated by the manipulation and faradization to cause an evacuation daily, a capsule containing ext. nux vomica $1 / 4$ gr., ox. gall. gr. $\mathrm{ij}$, aloin $\mathrm{I} / 6 \mathrm{gr}$., or something similar, should be administered at bedtime.

In spite of feeding, rubbing, and faradization, patients are occasionally found whose insomnia will persist. These cases I endeavor to control by giving them a hypnotic in such a way that they are not aware of the fact, and are led to attribute the sleep to the treatment. A favorite method is to saturate a loose vaginal tampon with a solution of chloral in glycerine and insert it the last thing at night. Sufficient chloral is absorbed to produce sleep, and the local anodyne effect upon the surrounding organs is not unpleasant. This can gradually be reduced in strength as the effect of the general treatment is sufficient to make it unnecessary. I have found also the triple valerianate pill of quinine, zinc and iron, as manufactured by W. H. Schieffelin \& Co., valuable in cases in this condition. Asafoetida pills are sometimes valuable at this point. The pills manufactured by Eli Lilli \& Co., of Indiana, conceal the taste and odor of this drug perfectly.

The urine should be examined occasionally in these cases to guard against harm arising from the excessive feeding.

If iron is indicated it can be given in small doses advantageously, in the fluid malt, or, in case malt is not an article of diet, in a capsule. For administration in malt citrate of iron, quinia, and strychnine in I-grain doses make an elegant preparation.

Success, however, depends much more upon the attendance the patient receives than upon the selection of drugs. Massage is given here with the idea of producing as much tissue change as possible, and the nurse who can accomplish the best results in this direction, as indicated by the amount of food taken and assimilated, is the greatest success. The faradization I do not usually intrust to a nurse, although an intelligent trained nurse can soon be taught to manipulate the faradic machine. The end sought here is simply to cause contraction of all the muscles of the body that can be brought under its influence, and to stimulate the circulation. For this purpose I place a large electrode under the two feet of the patient as she lies in bed with knees flexed; this electrode is attached to one pole of an ordinary interrupted faradic battery. At the other pole I attach a bifurcated cord terminating in two small hand electrodes, made to fit the palm of the hand in such a manner as not to interfere with the flexion of the fingers. A process of kneading or petrivage is performed over the surface of the body, dwelling particularly upon the motor points of the muscles, while the current is simply strong enough to produce an agreeable prickling sensation.

In this paper I have not been able to do more than to give the merest outline of a treatment of a condition which we all often meet in practice. In citing the particular case I have, I did so because of its being a typical one of the kind, and one in which the complete treatment in its most uncomplicated form could be demonstrated as a success. From this any intelligent physician can readily comprehend the scheme of treatment, and can as readily understand how many difficulties might arise that would complicate the treatment, at the same time not necessarily proving insurmountable barriers to its ultimate success.

163 State St.

\section{WATER CLOSETS AND PRIVY VAULTS.}

Abstract of a paper read before the Ohio State Sanitary Association, February 10, 1887 .

BY JOHN MCCURDY, M.D., OF YOUNGSTOWN, OHIO.

MEMBER OF OHIO STATE SANITARY ASSOCtation, MEMBER OF THE YOUNGSTOWN BOARD OF HEALTH, ETC.

To those who have given study to the above subjects they rank them first in importance. They 